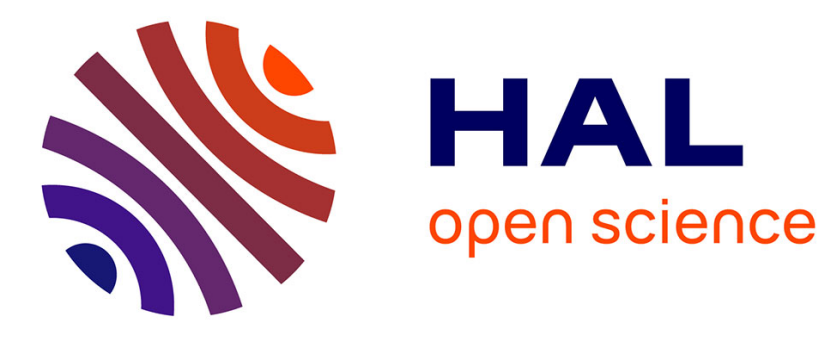

\title{
Pipeline following by visual servoing for Autonomous Underwater Vehicles
}

Guillaume Allibert, Minh-Duc Hua, Szymon Krupínski, Tarek Hamel

\section{To cite this version:}

Guillaume Allibert, Minh-Duc Hua, Szymon Krupínski, Tarek Hamel. Pipeline following by visual servoing for Autonomous Underwater Vehicles. Control Engineering Practice, 2019, 82, pp.151-160. hal-01920255

\section{HAL Id: hal-01920255 \\ https://hal.science/hal-01920255}

Submitted on 13 Nov 2018

HAL is a multi-disciplinary open access archive for the deposit and dissemination of scientific research documents, whether they are published or not. The documents may come from teaching and research institutions in France or abroad, or from public or private research centers.
L'archive ouverte pluridisciplinaire HAL, est destinée au dépôt et à la diffusion de documents scientifiques de niveau recherche, publiés ou non, émanant des établissements d'enseignement et de recherche français ou étrangers, des laboratoires publics ou privés. 


\title{
Pipeline following by visual servoing for Autonomous Underwater Vehicles
}

\author{
Guillaume Allibert ${ }^{\mathrm{a}, \mathrm{d}}$, Minh-Duc Hua ${ }^{\mathrm{a}}$, Szymon Krupínskib ${ }^{\mathrm{b}}$, Tarek Hamel ${ }^{\mathrm{a}, \mathrm{c}}$ \\ ${ }^{a}$ University of Côte d'Azur, CNRS, I3S, France. Emails: allibert(thamel, hua)@i3s.unice.fr \\ ${ }^{b}$ Cybernetix, Marseille, France. Email: szymon.krupinski@cybernetix.fr \\ ${ }^{c}$ Institut Universitaire de France, France \\ ${ }^{d}$ Corresponding author
}

\begin{abstract}
A nonlinear image-based visual servo control approach for pipeline following of fully-actuated Autonomous Underwater Vehicles (AUV) is proposed. It makes use of the binormalized Plücker coordinates of the pipeline borders detected in the image plane as feedback information while the system dynamics are exploited in a cascade manner in the control design. Unlike conventional solutions that consider only the system kinematics, the proposed control scheme accounts for the full system dynamics in order to obtain an enlarged provable stability domain. Control robustness with respect to model uncertainties and external disturbances is reinforced using integral corrections. Robustness and efficiency of the proposed approach are illustrated via both realistic simulations and experimental results on a real AUV.
\end{abstract}

Keywords: AUV, pipeline following, visual servoing, nonlinear control

\section{Introduction}

Underwater pipelines are widely used for transportation of oil, gas or other fluids from production sites to distribution sites. Laid down on the ocean floor, they are often subject to extreme conditions (temperature, pressure, humidity, sea current, vibration, salt, dust, etc.) that may lead to multiple problems such as corrosion, crack, joint failure, shock loading and leakage. Regular inspection, monitoring and maintenance of transportation pipelines are thus highly recommended for safe operation. Conventional pipeline monitoring and inspection methods generally consist in using surface ships and remotely operated underwater vehicles, with the consequence of slow response and mobilization time Christ and Wernli (2007). Moreover, methods involving human divers in deep water are difficult to implement due to the inhospitable environment with high health and safety risks. As underwater operations increase in scale and in complexity, the need for employing Autonomous Underwater Vehicles (AUV) increases Shukla and Karki (2016ba). However, unlike unmanned aerial vehicles that have seen an impressive growth within the last two decades, progress in AUV research and development has been drastically hindered by the lack of global positioning systems, particularly due to the attenuation of electromagnetic waves in water.

The dynamics of AUVs are very nonlinear, with highly coupled translational and rotational dynamics Fossen (2002); Leonard (1997). Strong perturbations due to sea currents are also a source of complexity. Robust control design for AUVs thus has been extensively investigated. However, existing control approaches such as PID Allen et al. (1997), LQR Naeem et al. (2003), $H_{\infty}$ Fryxell et al. (1996), optimal control Spangelo and Egeland (1994), sliding mode control Josserand (2006); Lapierre et al. (2008), Lyapunov backstepping-based control Repoulias and Papadopoulos (2007); Aguiar and Pascoal (2007); Antonelli (2007) and Lyapunov model-based control Refsnes et al. (2008); Smallwood and Whitcomb (2004) mostly concern the pre-programmed trajectory tracking problem with little regard to the local topography of the environment.

In this paper, the problem of pipeline following for AUVs, commonly addressed by using either a monocular camera or an acoustic sensor such as side scan sonar (SSS) or multi-beam echo-sounder, is revisited. Control objectives often consist in steering the vehicle above the pipeline and in regulating its forward speed to a reference value that can be specified in advance or online by a human operator. Most existing works on this topic have been devoted to pipeline detection from camera images or SSS-images and to the derivation of the relative heading and position (up to a scale factor) of the AUV with respect to (w.r.t.) the pipeline. Basic kinematic controllers have been applied without considering the system dynamics Matsumoto and Yoshihiko (1995); Antich and Ortiz (2003); Inzartsev and Pavin (2009); Bagnitsky et al. (2011) with the consequence that the stability is not systematically guaranteed. Other control approaches for pipeline following have been proposed in a more "abstract" manner in the sense that error tracking terms are directly defined from image features Rives and Borrelly (1997); Krupínski et al. (2012). These image-based visual servoing (IBVS) approaches do not require much knowledge about the $3 \mathrm{D}$ environment and demand less computations. For instance, Rives and Borrelly (1997) proposed an IBVS controller for fully-actuated AUVs using polar representation of lines (i.e. pipeline borders) while exploiting the so-called task-function approach developed by Samson et al. (1991). However, only local stability is proved since both the image Jacobian and Hes- 
sian matrices considered in the control design are evaluated at the desired pose in the image plane. The domain of convergence is thus impossible to be characterized. The present paper aims at extending the provable domain of stability by taking the vehicle dynamics into account and by adapting the IBVS control approach proposed in Mahony and Hamel (2005) to the case of AUVs. More precisely, image features used for control design are the bi-normalized Plücker coordinates Plücker (1865) of the pipeline borders. The resulting dynamic IBVS controller ensures the semi-global asymptotic stability.

This paper is organized as follows. Section 2 recalls notation and system modeling. In Section 3 , the problem of pipeline following by visual servoing is formulated. Section 4 presents the proposed controller based on a cascade inner-outer loop control architecture, where the inner-loop controller stabilizes the vehicle's velocities about a desired velocity setpoint and the outerloop controller derives the desired velocities and their derivative from image features. Convincing comparative simulation results and experimental validations, with a video as supplementary material, are reported in Section 5 to illustrate the robustness and performance of the proposed control approach. Finally, concluding remarks are provided in Section 6

Some basic materials of this paper such as notation and system modelling are borrowed from our other work Krupínski et al. (2017), which deals with a completely different IBVS control problem (i.e. fixed-point stabilization exploiting the homography matrix). Finally, for the sake of completeness in Section 4.2 the inner-loop controller is recalled from Krupínski et al. (2017). Note, however, that the main contribution of the present paper concerns the outer-loop control design level.

A primary version of this work has been presented in Krupínski et al. (2012) and a part of experimental results has been reported in Krupínski et al. (2015). A number of improvements w.r.t. Krupínski et al. (2012) are proposed in this paper. For instance, to mitigate the strong coupling between the vertical motion and the transverse and yaw motions of the AUV w.r.t. the pipeline that may lead to a large overshoot in altitude with a risk of collision with the ocean floor (see our prior work Krupínski et al. (2012)), a decoupling strategy has been introduced. Moreover, unlike Krupínski et al. (2012); Mahony and Hamel (2005) the desired feature is directly expressed in the body-fixed frame, avoiding the need of full attitude estimation to compute the visual error. Finally, the comparative simulation study w.r.t. the state-of-the-art IBVS controller Rives and Borrelly (1997) and the experimental validations have been newly developed.

\section{System Modelling}

\subsection{Notation}

The following notation is introduced (Fig. 1).

- Let $G$ and $B$ denote the AUV's center of mass (CoM) and center of buoyancy $(\mathrm{CoB})$, respectively. Let $m$ denote its mass and $\mathbf{J}_{0}$ denote its inertia matrix w.r.t. the CoB. $g$ denotes the gravity constant, i.e. $g \approx 9.81\left(\mathrm{~m} / \mathrm{s}^{2}\right)$.
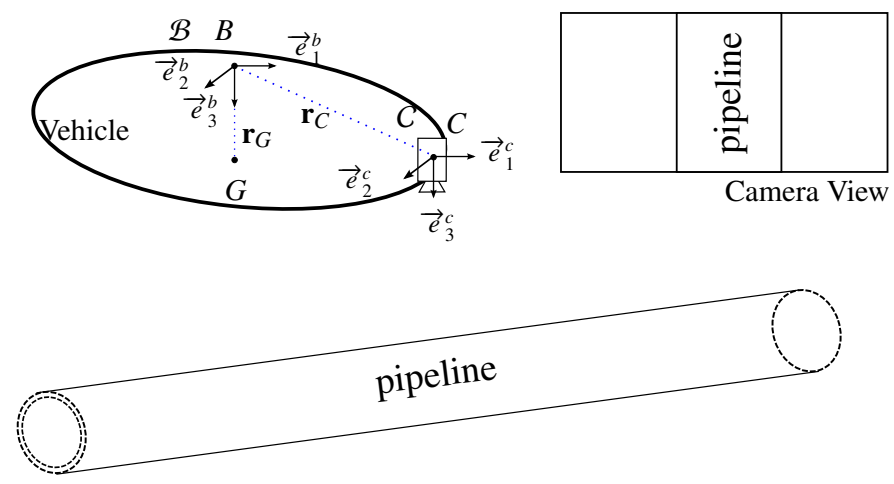

Figure 1: Notation

- $\mathcal{A}=\left\{O ; \vec{e}_{1}^{a}, \vec{e}_{2}^{a}, \vec{e}_{3}^{a}\right\}$ is an inertial frame. Let $\mathcal{B}=$ $\left\{B ; \vec{e}_{1}^{b}, \vec{e}_{2}^{b}, \vec{e}_{3}^{b}\right\}$ denote a frame attached to the AUV, with origin coinciding with the vehicle's CoB. Let $C=\left\{C ; \vec{e}_{1}^{c}, \vec{e}_{2}^{c}, \vec{e}_{3}^{c}\right\}$ be a frame attached to the camera, which is displaced from the origin of $\mathcal{B}$ by a vector $\overrightarrow{B C}$ and whose base vectors are parallel to those of $\mathcal{B}$. The vectors of coordinates expressed in $\mathcal{B}$ of $\overrightarrow{B C}$ and $\overrightarrow{B G}$ are denoted as $\mathbf{r}_{C} \in \mathbb{R}^{3}$ and $\mathbf{r}_{G} \in \mathbb{R}^{3}$, respectively.

- The orientation (i.e. attitude) of $\mathcal{B}$ w.r.t. $\mathcal{A}$ is represented by the rotation matrix $\mathbf{R} \in \mathrm{SO}(3)$. Let $\mathbf{p}$ and $\mathbf{p}_{C}$ denote the position of the origins of $\mathcal{B}$ and $C$ expressed in $\mathcal{A}$, respectively. One has $\mathbf{p}=\mathbf{p}_{C}-\mathbf{R r}_{C}$.

- The angular velocity vector of $\mathcal{B}$ relative to $\mathcal{A}$, expressed in $\mathcal{B}$, is denoted as $\boldsymbol{\Omega} \in \mathbb{R}^{3}$. The translational (or linear) velocity vectors of the origins of $\mathcal{B}$ and $C$, expressed in $\mathcal{B}$, are denoted as $\mathbf{V} \in \mathbb{R}^{3}$ and $\mathbf{V}_{C} \in \mathbb{R}^{3}$ respectively. One has $\mathbf{V}=\mathbf{V}_{C}-\boldsymbol{\Omega} \times \mathbf{r}_{C}$.

- The vector of coordinates of the fluid (i.e. current) velocity in $\mathcal{A}$ and $\mathcal{B}$ are denoted as $\mathbf{v}_{f}$ and $\mathbf{V}_{f}$, respectively. In this paper, it is assumed that $\mathbf{v}_{f}$ is constant. $\mathbf{V}_{h} \triangleq \mathbf{V}-\mathbf{V}_{f}$ is the vector of coordinates of the CoB's velocity w.r.t. the fluid.

- $\left\{\mathbf{e}_{1}, \mathbf{e}_{2}, \mathbf{e}_{3}\right\}$ denotes the canonical basis of $\mathbb{R}^{3}$. $\mathbf{I}_{3}$ is the identity matrix of $\mathbb{R}^{3 \times 3}$. For all $\mathbf{u} \in \mathbb{R}^{3}$, the notation $\mathbf{u}_{\times}$denotes the skew-symmetric matrix associated with the cross product by $\mathbf{u}$, i.e., $\mathbf{u}_{\times} \mathbf{v}=\mathbf{u} \times \mathbf{v}, \forall \mathbf{v} \in \mathbb{R}^{3} . \pi_{\mathbf{x}}=\mathbf{I}_{3}-\mathbf{x} \mathbf{x}^{\top}$ is the projection onto the tangent space of the sphere $S^{2}$ of a point $\mathbf{x} \in S^{2}$.

\subsection{Recall on system modelling}

Define $\mathbf{W}_{h} \triangleq\left[\mathbf{V}_{h}^{\top}, \mathbf{\Omega}^{\top}\right]^{\top} \in \mathbb{R}^{6}$. The total kinetic energy of the body-fluid system $E_{T}$ is defined as the sum of the kinetic energy of the vehicle $E_{B}$ and the one of the surrounding fluid $E_{F}$, i.e. $E_{T}=E_{B}+E_{F}$ with

$$
\begin{gathered}
E_{B}=\frac{1}{2} \mathbf{W}_{h}^{\top} \mathbf{M}_{B} \mathbf{W}_{h}, \text { with } \mathbf{M}_{B} \triangleq\left[\begin{array}{cc}
m \mathbf{I}_{3} & -m \mathbf{r}_{G \times} \\
m \mathbf{r}_{G \times} & \mathbf{J}_{0}
\end{array}\right] \\
E_{F}=\frac{1}{2} \mathbf{W}_{h}^{\top} \mathbf{M}_{A} \mathbf{W}_{h}, \text { with } \mathbf{M}_{A} \triangleq\left[\begin{array}{cc}
\mathbf{M}_{A}^{11} & \mathbf{M}_{A}^{12} \\
\mathbf{M}_{A}^{21} & \mathbf{M}_{A}^{22}
\end{array}\right]
\end{gathered}
$$

$\mathbf{M}_{A} \in \mathbb{R}^{6 \times 6}$ is referred to as the added mass matrix, which is approximately constant and symmetric Fossen (2002). Thus,

$$
E_{T}=\frac{1}{2} \mathbf{W}_{h}^{\top} \mathbf{M}_{T} \mathbf{W}_{h}, \text { with } \mathbf{M}_{T}=\left[\begin{array}{cc}
\mathbf{M} & \mathbf{D}^{\top} \\
\mathbf{D} & \mathbf{J}
\end{array}\right]
$$

with $\mathbf{M} \triangleq m \mathbf{I}_{3}+\mathbf{M}_{A}^{11}, \mathbf{J} \triangleq \mathbf{J}_{0}+\mathbf{M}_{A}^{22}, \mathbf{D} \triangleq m \mathbf{r}_{G \times}+\mathbf{M}_{A}^{21}$. The translational and rotational momentums are derived as 


$$
\left\{\begin{array}{l}
\boldsymbol{\Pi}_{t h}=\frac{\partial E_{T}}{\partial \mathbf{V}_{h}}=\mathbf{M} \mathbf{V}_{h}+\mathbf{D}^{\top} \boldsymbol{\Omega} \\
\mathbf{\Pi}_{r h}=\frac{\partial E_{T}}{\partial \boldsymbol{\Omega}}=\mathbf{J} \boldsymbol{\Omega}+\mathbf{D} \mathbf{V}_{h}
\end{array}\right.
$$

The equations of motion are given by Leonard (1997)

$$
\begin{aligned}
\dot{\mathbf{p}} & =\mathbf{R V} \\
\dot{\mathbf{R}} & =\mathbf{R} \boldsymbol{\Omega}_{\times} \\
\dot{\boldsymbol{\Pi}}_{t h} & =\boldsymbol{\Pi}_{t h} \times \mathbf{\Omega}+\mathbf{F}_{c}+\mathbf{F}_{g b}+\mathbf{F}_{d} \\
\dot{\boldsymbol{\Pi}}_{r h} & =\boldsymbol{\Pi}_{r h} \times \mathbf{\Omega}+\boldsymbol{\Pi}_{r h} \times \mathbf{V}_{h}+\boldsymbol{\Gamma}_{c}+\boldsymbol{\Gamma}_{g}+\boldsymbol{\Gamma}_{d}
\end{aligned}
$$

where $\mathbf{F}_{c} \in \mathbb{R}^{3}$ and $\boldsymbol{\Gamma}_{c} \in \mathbb{R}^{3}$ are the force and torque control vector inputs, $\mathbf{F}_{g b} \triangleq\left(m g-F_{b}\right) \mathbf{R}^{\top} \mathbf{e}_{3}$ is the sum of the gravitational and buoyancy forces, $\boldsymbol{\Gamma}_{g} \triangleq m g \mathbf{r}_{G} \times \mathbf{R}^{\top} \mathbf{e}_{3}$ is the gravity torque, and the hydrodynamic damping force and torque vectors $\mathbf{F}_{d}$ and $\boldsymbol{\Gamma}_{d}$ are modeled as

$$
\left\{\begin{array}{l}
\mathbf{F}_{d}\left(\mathbf{V}_{h}\right)=-\left(\mathbf{D}_{V l}+\left|\mathbf{V}_{h}\right| \mathbf{D}_{V q}\right) \mathbf{V}_{h} \\
\boldsymbol{\Gamma}_{d}(\boldsymbol{\Omega})=-\left(\mathbf{D}_{\Omega l}+|\boldsymbol{\Omega}| \mathbf{D}_{\Omega q}\right) \boldsymbol{\Omega}
\end{array}\right.
$$

with positive damping matrices $\mathbf{D}_{V l}, \mathbf{D}_{V q}, \mathbf{D}_{\Omega l}, \mathbf{D}_{\Omega q} \in \mathbb{R}^{3 \times 3}$.

\subsection{Model for control design}

The momentum terms $\boldsymbol{\Pi}_{t h}, \boldsymbol{\Pi}_{r h}$ and their dynamics (3c)-3d involve unknown current velocity $\mathbf{V}_{f}$, thereby complicating the control design process. Therefore, System (3) can be rewritten as follows

$$
\begin{aligned}
\dot{\mathbf{p}} & =\mathbf{R V} \\
\dot{\mathbf{R}} & =\mathbf{R} \boldsymbol{\Omega}_{\times} \\
\dot{\boldsymbol{\Pi}}_{t} & =\boldsymbol{\Pi}_{t} \times \mathbf{\Omega}+\mathbf{F}_{c}+\mathbf{F}_{g b}+\overline{\mathbf{F}}_{d}+\Delta_{F} \\
\dot{\mathbf{\Pi}}_{r} & =\boldsymbol{\Pi}_{r} \times \mathbf{\Omega}+\boldsymbol{\Pi}_{t} \times \mathbf{V}+\boldsymbol{\Gamma}_{c}+\boldsymbol{\Gamma}_{g}+\boldsymbol{\Gamma}_{d}+\boldsymbol{\Delta}_{\Gamma}
\end{aligned}
$$

with new momentum terms (compared to (2))

$$
\mathbf{\Pi}_{t} \triangleq \mathbf{M V}+\mathbf{D}^{\top} \mathbf{\Omega}, \mathbf{\Pi}_{r} \triangleq \mathbf{J} \boldsymbol{\Omega}+\mathbf{D V}
$$

and new dissipative force (compared to (4))

$$
\overline{\mathbf{F}}_{d} \triangleq-\left(\mathbf{D}_{V l}+|\mathbf{V}| \mathbf{D}_{V q}\right) \mathbf{V}
$$

and "disturbance" terms $\Delta_{F}$ and $\Delta_{\Gamma}$ given by

$$
\begin{gathered}
\left.\boldsymbol{\Delta}_{F} \triangleq-\mathbf{M} \boldsymbol{\Omega}_{\times} \mathbf{V}_{f}-(\mathbf{M V})_{f}\right)_{\times} \boldsymbol{\Omega}+\mathbf{F}_{d}-\overline{\mathbf{F}}_{d} \\
\boldsymbol{\Delta}_{\Gamma} \triangleq(\mathbf{M V})_{\times} \mathbf{V}_{f}-(\mathbf{M V})_{\times} \mathbf{V}-(\mathbf{M V})_{\times} \mathbf{V}_{f} \\
\left.-\mathbf{D} \boldsymbol{\Omega}_{\times} \mathbf{V}_{f}-(\mathbf{D V})_{f}\right)_{\times} \boldsymbol{\Omega}
\end{gathered}
$$

The disturbance terms $\boldsymbol{\Delta}_{F}$ and $\boldsymbol{\Delta}_{\Gamma}$ vanish if $\mathbf{v}_{f}=\mathbf{0}$. Otherwise, they should be addressed using either an estimator or integral compensation actions.

In the sequel the system's equations (5) will be used for control design, with the unknown disturbance terms $\Delta_{F}$ and $\Delta_{\Gamma}$ considered as constant vectors.

\section{Problem formulation of pipeline following by visual ser- voing}

Assume that the AUV is equipped with an Inertial Measurement Unit (IMU), a Doppler Velocity Log (DVL) and a monocular camera. The IMU provides measurements of the angular velocity $\boldsymbol{\Omega}$ and an approximate of the gravity direction $\mathbf{R}^{\top} \mathbf{e}_{3}$ (i.e., roll and pitch angles), whereas the DVL measures the translational velocity $\mathbf{V}$. The visual features considered are the pipeline borders assumed to be parallel to each other (see Fig. 2). Assume that the curvature of the pipeline is negligible so that the pipeline direction $\mathbf{u}$ in the inertial frame is approximatively constant. The inertial frame is chosen such that $\mathbf{u} \in \operatorname{span}\left(\mathbf{e}_{1}, \mathbf{e}_{3}\right)$.

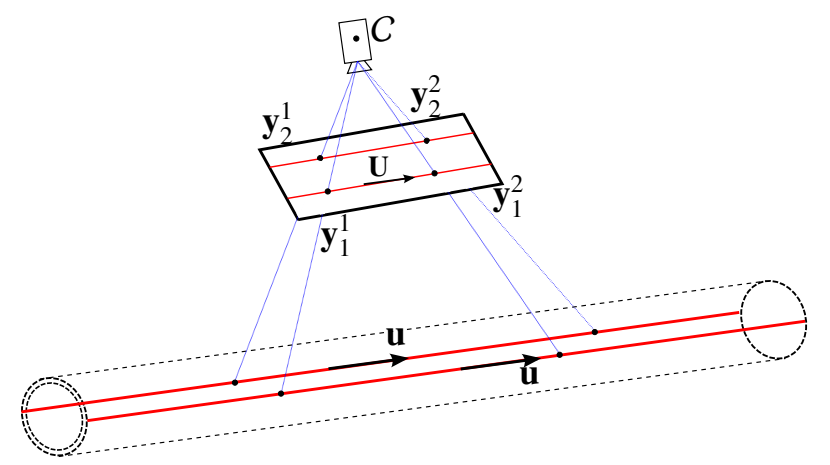

Figure 2: Geometrical basis of the pipeline-following visual servo control problem

Provided that the observed borderlines of the pipeline are parallel, their Plücker coordinates $\left(\mathbf{h}_{i}, \mathbf{U}\right) \in S^{2} \times S^{2}, i=\{1,2\}$, expressed in the camera frame $C$, can be measured directly from the image features Mahony and Hamel (2005) as follows (see Fig. 2)

$$
\left\{\begin{array}{l}
\mathbf{h}_{i} \triangleq \frac{\mathbf{y}_{i}^{1} \times \mathbf{y}_{i}^{2}}{\left|\mathbf{y}_{i}^{1} \times \mathbf{y}_{i}^{2}\right|} \\
\mathbf{U}= \pm \frac{\mathbf{h}_{1} \times \mathbf{h}_{2}}{\left|\mathbf{h}_{1} \times \mathbf{h}_{2}\right|}
\end{array}\right.
$$

where $\mathbf{y}_{i}^{1}$ and $\mathbf{y}_{i}^{2}$ are the metric pixel coordinates (i.e. 3D coordinates of a point divided by its depth) of points belonging to the observed borderline $i$ w.r.t. the optical center of the image. The direction of the pipeline $\mathbf{U}$ expressed in the camera frame is specified up to a sign that should be assigned by the operator. The proposed visual servo control is based on the centroid vector computed from visual features $(6)$ as follows (see Fig. 3)

$$
\mathbf{q} \triangleq \mathbf{h}_{1}+\mathbf{h}_{2}
$$

One verifies that $\mathbf{h}_{i}$ is also equal to $\mathbf{h}_{i}=\frac{\mathbf{H}_{i}}{\left|\mathbf{H}_{i}\right|}$ where $\mathbf{H}_{i}=\mathbf{P}_{i} \times \mathbf{U}$ and $\mathbf{P}_{i}$ is the vector of coordinates, expressed in $C$, of the closest point $P_{i}$ on the line to the origin of the camera frame $C$.

The kinematics of $\mathbf{U}, \mathbf{P}_{i}$ and $\mathbf{H}_{i}$, with $i=1,2$, are inherited from the camera motion relative to the observed pipeline. Since $\mathbf{u}$ is constant by assumption, one obtains Mahony and Hamel (2005)

$$
\left\{\begin{aligned}
\dot{\mathbf{U}} & =-\boldsymbol{\Omega} \times \mathbf{U} \\
\dot{\mathbf{P}}_{i} & =-\mathbf{\Omega} \times \mathbf{P}_{i}-\pi_{\mathbf{U}} \mathbf{V}_{C} \\
\dot{\mathbf{H}}_{i} & =-\boldsymbol{\Omega} \times \mathbf{H}_{i}-\mathbf{V}_{C} \times \mathbf{U}
\end{aligned}\right.
$$

From these equations one derives the dynamics of the centroid vector $\mathbf{q}$ as

$$
\dot{\mathbf{q}}=-\mathbf{\Omega} \times \mathbf{q}-\mathbf{Q}\left(\mathbf{V}_{C} \times \mathbf{U}\right)
$$




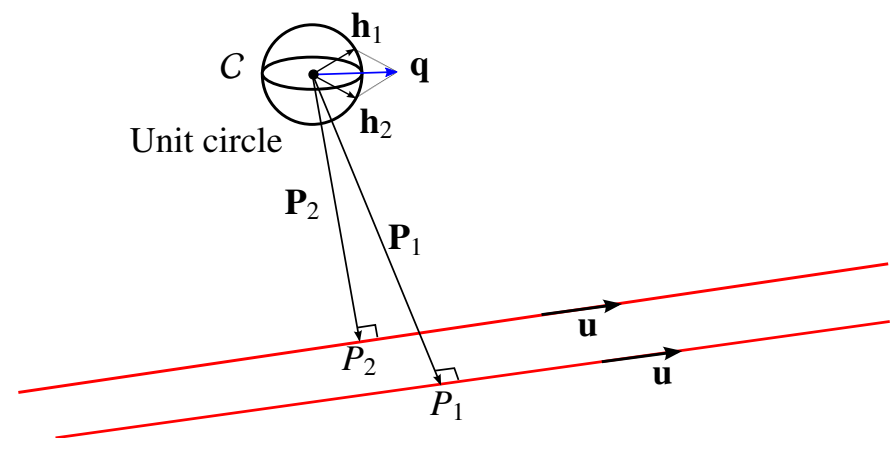

Figure 3: Illustration of the construction of the visual variable $\mathbf{q}$

where $\mathbf{Q} \triangleq \sum_{i=1}^{2} \frac{1}{\left|\mathbf{H}_{i}\right|} \pi_{\mathbf{h}_{i}}$ is a symmetric positive definite matrix, with $\left|\mathbf{H}_{i}\right|$ equal to the distance from the origin of the camera frame to the borderline of index $i$, i.e. $\left|\mathbf{H}_{i}\right|=\left|\mathbf{P}_{i}\right|$. Since these distances are not known when using a monocular camera, the matrix $\mathbf{Q}$ is not known either for control design.

Let $\mathbf{q}^{\star}$ be the reference value of $\mathbf{q}$. Control action must ensure the asymptotic stabilization of $\mathbf{q}$ about $\mathbf{q}^{\star}$. The latter is typically chosen constant and parallel to $\mathbf{e}_{2}$ (i.e. $\dot{\mathbf{q}}^{\star}=\mathbf{0}$, $\left.\mathbf{q}^{\star}=\left|\mathbf{q}^{\star}\right| \mathbf{e}_{2}\right)$, leading implicitly to stabilize the AUV in the middle of the pipeline at the desired relative distance encoded in $\left|\mathbf{q}^{\star}\right|$.

Remark 1. It is worth providing a physical interpretation on the magnitude of $\mathbf{q}^{\star}$. It is verified that $\left|\mathbf{q}^{\star}\right|=2 \cos \alpha^{\star}$ with $\alpha^{\star}=\operatorname{artan}\left(l_{p} /\left(2 d^{\star}\right)\right)$, where $l_{p}$ and $d^{\star}$ are respectively the width of the pipeline and the distance between the camera and the pipeline associated to $\mathbf{q}^{\star}$. This means that the norm of $\mathbf{q}^{\star}$ must be chosen smaller than 2 (i.e. $\left|\mathbf{q}^{\star}\right|<2$ ) and that the more it gets close to 2 the larger the distance $d^{\star}$ (i.e., $\left.\lim _{\left|\mathbf{q}^{\star}\right| \rightarrow 2} d^{\star}=+\infty\right)$.

Define the visual position-like error as

$$
\boldsymbol{\delta}_{1} \triangleq \mathbf{q}-\pi_{\mathbf{U}} \mathbf{q}^{\star}
$$

Note that $\boldsymbol{\delta}_{1}$ is orthogonal to $\mathbf{U}$, which is an important property to be exploited in the outer-loop control design.

The control objective consists of stabilizing the lateral and vertical positions of the vehicle w.r.t. the pipeline to the desired values with null roll angle (i.e. stabilizing $\delta_{1}$ and $\phi$ about zero), stabilizing the vector $\mathbf{U}$ about $\mathbf{e}_{1}$, and $\mathbf{V}^{\top} \mathbf{U}$ about the reference speed $v_{r} \in \mathbb{R}$. Additionally, the pitch angle must asymptotically converge to the slope angle $\beta(-\pi / 2<\beta<\pi / 2)$ of the pipeline.

Lemma 1. If ( $\left.\mathbf{U}, \mathbf{e}_{2}^{\top} \mathbf{R}^{\top} \mathbf{e}_{3}\right)$ asymptotically converge to $\left(\mathbf{e}_{1}, 0\right)$, then roll, pitch and yaw angles locally asymptotically converge to $(0, \beta, 0)$.

Proof. Since $\mathbf{u} \in \operatorname{span}\left(\mathbf{e}_{1}, \mathbf{e}_{3}\right)$ and under assumption that $\mathbf{U} \rightarrow$ $\mathbf{e}_{1}$ and $\mathbf{e}_{2}^{\top} \mathbf{R}^{\top} \mathbf{e}_{3} \rightarrow 0$, one deduces $\sin \phi \rightarrow 0, \sin \theta \rightarrow \sin \beta$, $\sin \psi \rightarrow 0$, which locally ensures the convergence of $(\phi, \theta, \psi)$ to $(0, \beta, 0)$.

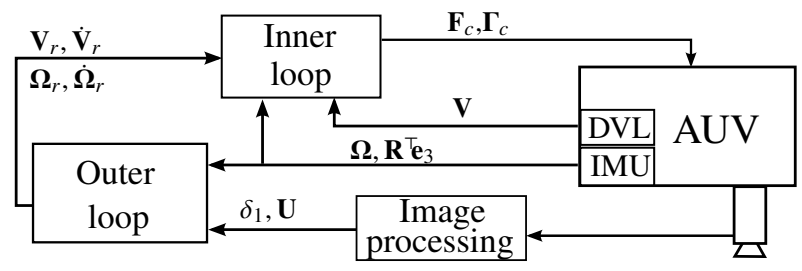

Figure 4: Block diagram of the proposed control scheme

\section{Control design}

The following cascade inner-outer loop control architecture (illustrated by Fig. 4) is adopted.

- The inner-loop control defines the force and torque control vectors $\mathbf{F}_{c}$ and $\boldsymbol{\Gamma}_{c}$ that ensure the asymptotic stabilization of $(\mathbf{V}, \boldsymbol{\Omega})$ about $\left(\mathbf{V}_{r}, \boldsymbol{\Omega}_{r}\right)$, where the reference velocities $\mathbf{V}_{r}$ and $\boldsymbol{\Omega}_{r}$ are defined by the outer-loop control.

- The outer-loop control is specifically designed from the image features to define the desired velocity setpoint $\mathbf{V}_{r}$ and $\boldsymbol{\Omega}_{r}$ as well as their derivative to fulfill the main objective of stabilizing $\left(\boldsymbol{\delta}_{1}, \mathbf{U}, \mathbf{V}\right)$ about $\left(\mathbf{0}, \mathbf{e}_{1}, v_{r} \mathbf{e}_{1}\right)$.

\subsection{Outer-loop control design}

For a fully-actuated AUV with force and torque control inputs, it is not too difficult to design an inner-loop controller that ensures the global asymptotic stability and local exponential stability of the equilibrium $(\mathbf{V}, \boldsymbol{\Omega})=\left(\mathbf{V}_{r}, \boldsymbol{\Omega}_{r}\right)$, provided that the derivatives of $\mathbf{V}_{r}$ and $\boldsymbol{\Omega}_{r}$ are computable by the controller. Let us thus postpone the inner-loop control design and focus on the outer-loop control design, which is the main contribution of this paper.

The outer-loop control design is directly based on the features measured in the image plane, with the objective of stabilizing $\left(\boldsymbol{\delta}_{1}, \mathbf{U}, \mathbf{V}\right)$ about $\left(\mathbf{0}, \mathbf{e}_{1}, v_{r} \mathbf{e}_{1}\right)$.

From (7), (8) and (9) one verifies that the dynamics of $\delta_{1}$ satisfies

$$
\begin{gathered}
\dot{\boldsymbol{\delta}}_{1}=-\mathbf{\Omega} \times \mathbf{q}-\mathbf{Q}\left(\mathbf{V}_{C} \times \mathbf{U}\right)+\left(\dot{\mathbf{U}} \mathbf{U}^{\top}+\mathbf{U} \dot{\mathbf{U}}^{\top}\right) \mathbf{q}^{\star} \\
=-\mathbf{\Omega} \times\left(\boldsymbol{\delta}_{1}+\pi_{\mathbf{U}} \mathbf{q}^{\star}\right)-\mathbf{Q}\left(\mathbf{V}_{C} \times \mathbf{U}\right)+ \\
\left(-\mathbf{\Omega}_{\times} \mathbf{U} \mathbf{U}^{\top}+\mathbf{U} \mathbf{U}^{\top} \boldsymbol{\Omega}_{\times}\right) \mathbf{q}^{\star} \\
=-\mathbf{\Omega} \times \boldsymbol{\delta}_{1}-\pi_{\mathbf{U}}\left(\mathbf{\Omega} \times \mathbf{q}^{\star}\right)-\mathbf{Q}\left(\mathbf{V}_{C} \times \mathbf{U}\right)
\end{gathered}
$$

Now in order to provide the reader with some control insights, the kinematic case using the velocities $\mathbf{V}$ and $\boldsymbol{\Omega}$ as control inputs is investigated.

Lemma 2. (Kinematic Control) The kinematic controller

$$
\left\{\begin{array}{l}
\boldsymbol{\Omega}=k_{u} \mathbf{e}_{1} \times \mathbf{U} \\
\mathbf{V}=\mathbf{U} \times \boldsymbol{\delta}_{1}+v_{r} \mathbf{U}+\boldsymbol{\Omega} \times \mathbf{r}_{C}
\end{array}\right.
$$

with $k_{u}$ a positive gain, globally asymptotically stabilize $\mathbf{U}$ about $\pm \mathbf{e}_{1}$ and $\boldsymbol{\delta}_{1}$ about zero. Additionally, the velocities $\mathbf{\Omega}$ and $\mathbf{V}$ converge to zero and $v_{r} \mathbf{U}$, respectively. 
Proof. Consider the following positive storage function:

$$
\mathcal{S}_{1} \triangleq 1-\mathbf{U}^{\top} \mathbf{e}_{1}
$$

From (7), 11a and (12) one verifies that the derivative of $\mathcal{S}_{1}$ satisfies

$$
\dot{\mathcal{S}}_{1}=-\mathbf{U}^{\top} \boldsymbol{\Omega}_{\times} \mathbf{e}_{1}=-\boldsymbol{\Omega}^{\top}\left(\mathbf{e}_{1} \times \mathbf{U}\right)=-k_{u}\left(U_{2}^{2}+U_{3}^{2}\right)
$$

Provided that $\boldsymbol{\Omega}$ is considered as control input, system (7) is autonomous. Therefore, the application of LaSalle's principle ensures the convergence of $\dot{\mathcal{S}}_{1}$ and thus, of $U_{2}$ and $U_{3}$ to zero. This implies that $\mathbf{U}$ converges to either $\mathbf{e}_{1}$ or $-\mathbf{e}_{1}$. The convergence of $\boldsymbol{\Omega}$ to zero then follows from its definition (11a).

From $111 \mathrm{~b}$ ) one deduces $\mathbf{V}_{C}=k_{\delta} \mathbf{U} \times \boldsymbol{\delta}_{1}+v_{r} \mathbf{U}$. Now, consider the second positive storage function $\mathcal{S}_{2} \triangleq \frac{1}{2}\left|\boldsymbol{\delta}_{1}\right|^{2}$. Using (10), and the expression of $\mathbf{V}_{C}$ obtained previously and the orthogonality of $\boldsymbol{\delta}_{1}$ to $\mathbf{U}$, one deduces

$$
\begin{aligned}
\dot{\mathcal{S}}_{2} & =-\boldsymbol{\delta}_{1}^{\top} \pi_{\mathbf{U}}\left(\boldsymbol{\Omega} \times \mathbf{q}^{\star}\right)-\boldsymbol{\delta}_{1}^{\top} \mathbf{Q}\left(\mathbf{V}_{C} \times \mathbf{U}\right) \\
& =-\boldsymbol{\delta}_{1}^{\top} \pi_{\mathbf{U}}\left(\boldsymbol{\Omega} \times \mathbf{q}^{\star}\right)+k_{\delta} \boldsymbol{\delta}_{1}^{\top} \mathbf{Q}\left(\mathbf{U} \times\left(\mathbf{U} \times \boldsymbol{\delta}_{1}\right)\right) \\
& =-\boldsymbol{\delta}_{1}^{\top} \pi_{\mathbf{U}}\left(\boldsymbol{\Omega} \times \mathbf{q}^{\star}\right)-k_{\delta} \boldsymbol{\delta}_{1}^{\top} \mathbf{Q} \boldsymbol{\delta}_{1}
\end{aligned}
$$

Since the matrix $\mathbf{Q}$ is positive definite and the vanishing term $\pi_{\mathbf{U}}\left(\boldsymbol{\Omega} \times \mathbf{q}^{\star}\right)$ remains bounded for all time, one deduces from (18) and the definition of $\mathcal{S}_{2}$ that $\dot{\mathcal{S}}_{2}$ and, thus, $\delta_{1}$ converge to zero. Finally, the convergence of $\boldsymbol{\delta}_{1}$ and $\boldsymbol{\Omega}$ to zero ensure the convergence of $\mathbf{V}$ to $v_{r} \mathbf{U}$.

Remark 2. Since $\mathbf{V}$ and $\mathbf{\Omega}$ are not the physical control variables, some modifications should be made. In view of Lemma2 one may define the reference velocities $\mathbf{V}_{r}$ and $\mathbf{\Omega}_{r}$ as in the right hand side of Eqs. (11a)-111b) and apply an inner-loop control to ensure that $\mathbf{V}$ and $\mathbf{\Omega}$ converge to $\mathbf{V}_{r}$ and $\mathbf{\Omega}_{r}$. However, since the derivative of $\mathbf{V}_{r}$ is not computable by the inner-loop control due to the term $\delta_{1}$ involved in the expression (11b) and, subsequently, the stability of the equilibrium $(\mathbf{V}, \mathbf{\Omega})=\left(\mathbf{V}_{r}, \mathbf{\Omega}_{r}\right)$ is no longer guaranteed unconditionally. More precisely, in order to compute the derivative of $\mathbf{V}_{r}$, one needs to know the derivative of $\boldsymbol{\delta}_{1}$. Nevertheless, in view of the expression (10) of $\dot{\boldsymbol{\delta}}_{1}$, it is not computable by the controller due to the unknown matrix $\mathbf{Q}$.

As mentioned previously, the knowledge of the derivative terms $\dot{\mathbf{V}}_{r}$ and $\dot{\boldsymbol{\Omega}}_{r}$ is required by the inner-loop controller. To this purpose, the reference velocities $\mathbf{V}_{r}$ and $\boldsymbol{\Omega}_{r}$ are defined as (compared to $(11 \mathrm{a})-(11 \mathrm{~b})$

$$
\left\{\begin{array}{l}
\mathbf{\Omega}_{r} \triangleq k_{u} \mathbf{e}_{1} \times \mathbf{U}-k_{\omega} \mathbf{e}_{1}\left(\mathbf{e}_{2}^{\top} \mathbf{R}^{\top} \mathbf{e}_{3}\right) \\
\mathbf{V}_{r} \triangleq\left[\mathbf{e}_{1}\right]_{\times}\left[\begin{array}{c}
0 \\
\overline{\boldsymbol{\delta}}_{2}
\end{array}\right]+v_{r} \mathbf{e}_{1}+\boldsymbol{\Omega}_{r} \times \mathbf{r}_{C}
\end{array}\right.
$$

where $k_{u}$ and $k_{\omega}$ are some positive gains, and the augmented variable $\overline{\boldsymbol{\delta}}_{2} \in \mathbb{R}^{2}$ is the solution to the augmented system:

$$
\dot{\overline{\boldsymbol{\delta}}}_{2}=\mathbf{K}_{1} \overline{\boldsymbol{\delta}}_{1}-\mathbf{K}_{2} \overline{\boldsymbol{\delta}}_{2}, \overline{\boldsymbol{\delta}}_{2}(0)=\overline{\boldsymbol{\delta}}_{0}
$$

with $\overline{\boldsymbol{\delta}}_{0} \in \mathbb{R}^{2}$ the initial condition, some positive diagonal $2 \times 2$ gain matrices $\mathbf{K}_{1}=\operatorname{diag}\left(k_{11}, k_{12}\right), \mathbf{K}_{2}=\operatorname{diag}\left(k_{21}, k_{22}\right) \in \mathbb{R}^{2 \times 2}$, and $\bar{\delta}_{1} \triangleq\left[\delta_{1,2}, \delta_{1,3}\right]^{\top} \in \mathbb{R}^{2}$ the vector of the two last components of $\boldsymbol{\delta}_{1}$. Since the derivative of $\mathbf{U}$ and $\overline{\boldsymbol{\delta}}_{2}$, given by (7) and (15) respectively, can be computed by the controller and since $\boldsymbol{\delta}_{2}$ can be obtained by integration of Eq. (15), it is straightforward to verify that $\dot{\mathbf{V}}_{r}$ and $\dot{\boldsymbol{\Omega}}_{r}$ are also computable by the controller.

Proposition 1. Let the reference velocities $\mathbf{V}_{r}$ and $\mathbf{\Omega}_{r}$ be specified by the outer-loop controller as in Eqs. (14a)-(14b). Apply any inner-loop controller that ensures the global asymptotic stability and local exponential stability of the equilibrium $\left(\mathbf{V}_{C}, \mathbf{\Omega}\right)=\left(\mathbf{V}_{C r}, \boldsymbol{\Omega}_{r}\right)$. Let $\lambda_{\overline{\mathbf{Q}}}^{\text {sup }}, \lambda_{\overline{\mathbf{Q}}}^{\text {inf }}>0$ denote the supremum of the largest eigenvalue and the infimum of the smallest eigenvalue of the symmetric positive definite matrix $\overline{\mathbf{Q}} \triangleq$ $\left[\begin{array}{ll}Q_{2,2} & Q_{2,3} \\ Q_{2,3} & Q_{3,3}\end{array}\right] \in \mathbb{R}^{2 \times 2}$. Let $\gamma_{\dot{\overline{\mathbf{Q}}}}$ denote the bound of $\dot{\overline{\mathbf{Q}}}$. Assume that the control gains $\mathbf{K}_{1}$ and $\mathbf{K}_{2}$ involved in Eqs. (14b) and (15) satisfy

$$
k_{1 \max }<\frac{k_{2 \min }^{2}}{\varepsilon \lambda_{\overline{\mathbf{Q}}}^{\text {sup }}}, \quad k_{1 \min }>\frac{(1+\varepsilon) \gamma_{\dot{\mathbf{Q}}}}{2 \varepsilon\left(\lambda_{\overline{\mathbf{Q}}}^{\text {inf }}\right)^{2}} k_{2 \max }
$$

with some positive number $\varepsilon$ and

$$
\begin{array}{ll}
k_{1 \max } \triangleq \max \left(k_{11}, k_{12}\right), & k_{1 \min } \triangleq \min \left(k_{11}, k_{12}\right) \\
k_{2 \max } \triangleq \max \left(k_{21}, k_{22}\right), & k_{2 \min } \triangleq \min \left(k_{21}, k_{22}\right)
\end{array}
$$

Then, $\mathbf{U}$ is stabilized about $\pm \mathbf{e}_{1}$ and $\boldsymbol{\delta}_{1}$ and $\overline{\boldsymbol{\delta}}_{2}$ are stabilized about zero. Additionally, $(\mathbf{\Omega}, \mathbf{V})$ asymptotically converge to $\left(\mathbf{0}, v_{r} \mathbf{U}\right)$.

Proof. As a consequence of the inner-loop control, the velocity errors $\widetilde{\mathbf{V}} \triangleq \mathbf{V}-\mathbf{V}_{r}$ and $\widetilde{\mathbf{\Omega}} \triangleq \boldsymbol{\Omega}-\mathbf{\Omega}_{r}$ converge to zero.

First, the convergence of $U_{2}$ and $U_{3}$ to zero is studied. Consider the storage function $\mathcal{S}_{1}$ defined by (12). One verifies that

$$
\dot{\mathcal{S}}_{1}=\mathbf{\Omega}^{\top}\left(\mathbf{e}_{1} \times \mathbf{U}\right)=\left(\widetilde{\mathbf{\Omega}}+\mathbf{\Omega}_{r}\right)^{\top}\left(\mathbf{e}_{1} \times \mathbf{U}\right)=-k_{u} U_{2}^{2}-k_{u} U_{3}^{2}+\varepsilon_{\mathcal{S}_{1}}
$$

with $\varepsilon_{\mathcal{S}_{1}} \triangleq \widetilde{\mathbf{\Omega}}^{\top}\left(\mathbf{e}_{1} \times \mathbf{U}\right)$ a vanishing term. The application of Barbalat's lemma (see Khalil (2002)) then ensures the convergence of $U_{2}$ and $U_{3}$ to zero, which implies that $\mathbf{U}$ converge to either $\mathbf{e}_{1}$ or $-\mathbf{e}_{1}$.

Now the convergence of $\boldsymbol{\Omega}_{r}$ to zero is studied. Consider the storage function $\mathcal{S}_{3}=1-\mathbf{e}_{3}^{\top} \mathbf{R}^{\top} \mathbf{e}_{3}$. One verifies that

$$
\begin{aligned}
\dot{\mathcal{S}}_{3} & =-\left(\widetilde{\mathbf{\Omega}}+\mathbf{\Omega}_{r}\right)^{\top}\left(\mathbf{e}_{3} \times \mathbf{R}^{\top} \mathbf{e}_{3}\right) \\
& =-k_{\omega}\left(\mathbf{e}_{2}^{\top} \mathbf{R}^{\top} \mathbf{e}_{3}\right) \mathbf{e}_{1}^{\top}\left(\mathbf{e}_{3} \times \mathbf{R}^{\top} \mathbf{e}_{3}\right)+\varepsilon_{\mathcal{S}_{3}} \\
& =-k_{\omega}\left|\mathbf{e}_{2}^{\top} \mathbf{R}^{\top} \mathbf{e}_{3}\right|^{2}+\varepsilon_{\mathcal{S}_{3}}
\end{aligned}
$$

with $\varepsilon_{\mathcal{S}_{3}} \triangleq-k_{u}\left(\mathbf{e}_{1} \times \mathbf{U}\right)^{\top}\left(\mathbf{e}_{3} \times \mathbf{R}^{\top} \mathbf{e}_{3}\right)-\widetilde{\mathbf{\Omega}}^{\top}\left(\mathbf{e}_{3} \times \mathbf{R}^{\top} \mathbf{e}_{3}\right)$ a vanishing term. From there the application of Barbalat's lemma ensures the convergence of $\mathbf{e}_{2}^{\top} \mathbf{R}^{\top} \mathbf{e}_{3}$ to zero. One then easily deduces the convergence of $\boldsymbol{\Omega}$ and $\boldsymbol{\Omega}_{r}$ to zero using its definition (14a).

The convergence of $\boldsymbol{\delta}_{1}$ and $\overline{\boldsymbol{\delta}}_{2}$ to zero is now investigated. Using (10), (14b) and (15) one deduces

$$
\dot{\delta}_{1}=\mathbf{Q}\left(\mathbf{U} \times \mathbf{V}_{r}\right)+\boldsymbol{\epsilon}_{\delta 1}=-\mathbf{Q} \pi_{\mathbf{e}_{1}}\left[\begin{array}{c}
0 \\
\overline{\boldsymbol{\delta}}_{2}
\end{array}\right]+\boldsymbol{\epsilon}_{\delta 1}=-\mathbf{Q}\left[\begin{array}{c}
0 \\
\overline{\boldsymbol{\delta}}_{2}
\end{array}\right]+\boldsymbol{\epsilon}_{\delta 1}
$$


with $\boldsymbol{\epsilon}_{\delta 1} \triangleq-\boldsymbol{\Omega}_{\times} \boldsymbol{\delta}_{1}-\pi_{\mathbf{U}}\left(\boldsymbol{\Omega} \times \mathbf{q}^{\star}\right)+\mathbf{Q}\left(\mathbf{U} \times\left(\widetilde{\mathbf{V}}+\mathbf{\Omega} \times \mathbf{r}_{C}\right)\right)$ a vanishing term. One notes that the first component of $\boldsymbol{\delta}_{1}$ converges to zero by construction since $\delta_{1}$ is orthogonal to $\mathbf{U}$.

One deduces the following zero-dynamics, corresponding to $\boldsymbol{\epsilon}_{\delta_{1}} \equiv \mathbf{0}$ :

$$
\left\{\begin{array}{l}
\dot{\overline{\boldsymbol{\delta}}}_{1}=-\overline{\mathbf{Q}} \overline{\boldsymbol{\delta}}_{2} \\
\dot{\overline{\boldsymbol{\delta}}}_{2}=\mathbf{K}_{1} \overline{\boldsymbol{\delta}}_{1}-\mathbf{K}_{2} \overline{\boldsymbol{\delta}}_{2}
\end{array}\right.
$$

By application of singular perturbation theory Khalil (2002), in order to prove the convergence of $\boldsymbol{\delta}_{1}$ and $\overline{\boldsymbol{\delta}}_{2}$ to zero, it suffices to prove the exponential stability of the equilibrium $\left(\boldsymbol{\delta}_{1}, \overline{\boldsymbol{\delta}}_{2}\right)=$ $(\mathbf{0 , 0})$ of the zero-dynamics 20$]$.

Consider the following Lyapunov function candidate:

$$
\begin{aligned}
\mathcal{L} & =\frac{1+\varepsilon}{2} \overline{\boldsymbol{\delta}}_{1}^{\top} \overline{\mathbf{Q}}^{-1} \overline{\boldsymbol{\delta}}_{1}+\frac{1}{2} \overline{\boldsymbol{\delta}}_{2}^{\top} \mathbf{K}_{1}^{-1} \overline{\boldsymbol{\delta}}_{2}-\varepsilon \overline{\boldsymbol{\delta}}_{1}^{\top} \mathbf{K}_{2}^{-1} \overline{\boldsymbol{\delta}}_{2} \\
& \geq \frac{1+\varepsilon}{2 \lambda_{\overline{\mathbf{Q}}}^{\text {sup }}}\left|\overline{\boldsymbol{\delta}}_{1}\right|^{2}+\frac{1}{2 k_{1 \max }}\left|\overline{\boldsymbol{\delta}}_{2}\right|^{2}-\frac{\varepsilon}{k_{2 \min }}\left|\overline{\boldsymbol{\delta}}_{1}\right|\left|\overline{\boldsymbol{\delta}}_{2}\right|
\end{aligned}
$$

with some positive number $\varepsilon$. One verifies from (20) and 21) that

$$
\begin{aligned}
\dot{\mathcal{L}}= & \frac{1+\varepsilon}{2} \overline{\boldsymbol{\delta}}_{1}^{\top} \overline{\mathbf{Q}}^{-1} \dot{\overline{\mathbf{Q}}} \overline{\mathbf{Q}}^{-1} \overline{\boldsymbol{\delta}}_{1}-\overline{\boldsymbol{\delta}}_{2}^{\top} \mathbf{K}_{1}^{-1} \mathbf{K}_{2} \overline{\boldsymbol{\delta}}_{2}-\varepsilon \overline{\boldsymbol{\delta}}_{1}^{\top} \mathbf{K}_{2}^{-1} \mathbf{K}_{1} \overline{\boldsymbol{\delta}}_{1} \\
& +\varepsilon \overline{\boldsymbol{\delta}}_{2}^{\top} \mathbf{K}_{2}^{-1} \overline{\mathbf{Q}} \overline{\boldsymbol{\delta}}_{2} \\
\leq & -\left(\frac{\varepsilon k_{1 \min }}{k_{2 \max }}-\frac{(1+\varepsilon) \gamma_{\dot{\mathbf{Q}}}}{2\left(\lambda_{\overline{\mathbf{Q}}}^{\text {inf }}\right)^{2}}\right)\left|\overline{\boldsymbol{\delta}}_{1}\right|^{2}-\left(\frac{k_{2 \min }}{k_{1 \max }}-\frac{\varepsilon \lambda_{\overline{\mathbf{Q}}}^{\text {sup }}}{k_{2 \min }}\right)\left|\overline{\boldsymbol{\delta}}_{2}\right|^{2}
\end{aligned}
$$

From there, using condition (16) one deduces that $\mathcal{L}$ is positive definite and $\dot{\mathcal{L}}$ negative definite. The exponential convergence of $\bar{\delta}_{1}$ and $\bar{\delta}_{2}$ to zero then directly follows, allowing one to conclude the proof.

Remark 3. The outer-loop controller (14)-(15) has been improved w.r.t. the one proposed in our prior work Krupinski et al. (2012). In particular, the use of diagonal gain matrices $\mathbf{K}_{1}$ and $\mathbf{K}_{2}$ (justified by rigourous stability analysis) instead of the corresponding scalar gains used in Krupinski et al. (2012) allows one to locally decouple the outer-loop system (in first order approximations) into 3 independent subsystems corresponding to yaw, vertical and lateral dynamics, with the flexibility of independent gain tuning. This allows one to limit the influence of yaw and lateral dynamics on the transient behaviour of the vertical motion and thus avoid large overshoot in the altitude and limit the risk of collision with the ocean floor.

An additional modification to the outer-loop controller (14)(15) in order to reduce the influence of a large initial yaw error on the transient translational motion can be made by replacing the expression (14b) by the following equation:

$$
\mathbf{V}_{r}=\mu\left(\left|U_{1}\right|\right)\left[\mathbf{e}_{1}\right]_{\times}\left[\begin{array}{c}
0 \\
\overline{\boldsymbol{\delta}}_{2}
\end{array}\right]+\mu\left(\left|U_{1}\right|\right) v_{r} \mathbf{e}_{1}+\boldsymbol{\Omega}_{r} \times \mathbf{r}_{C}
$$

where $\mu(\cdot)$ is a differentiable monotonic increasing function defined in $[0,1]$ satisfying $\mu(0)>0$ and $\mu(1)=1$. For instance, $\mu(x)=\varepsilon+(1-\varepsilon) x^{2 n}$, with $0<\varepsilon<1$ a small number and $n$ a large integer, has been chosen in the simulation section.
The introduction of the function $\mu(\cdot)$ allows one to prioritize the stabilization of $\mathbf{U}$ to $\mathbf{e}_{1}$ over the stabilization of other control variables (i.e. $\delta_{1}$ and $V_{1}$ ). It can be easily shown that this modification does not affect the stability results stated in Proposition 1. In fact, from the proof of Proposition 1 one notes that the outer-loop control (14a) of $\boldsymbol{\Omega}_{r}$ ensures the convergence of $\mathbf{U}$ to $\pm \mathbf{e}_{1}$ independently from any expression of $\mathbf{V}_{r}$. Therefore, $\mu(\cdot)$ ultimately converges to 1 , which implies that 22 is ultimately equivalent to $14 \mathrm{~b}$ and hence the associated stability analysis can proceed identically.

\subsection{Recall on inner-loop control design}

Although the inner-loop control design for a fully-actuated AUV is not too challenging and is not the main preoccupation of this paper, it is recalled here for completeness.

The inner-loop control objective can be stated as the stabilization of $(\widetilde{\mathbf{V}}, \widetilde{\mathbf{\Omega}})$ about zero, with $\widetilde{\mathbf{V}} \triangleq \mathbf{V}-\mathbf{V}_{r}$ and $\widetilde{\mathbf{\Omega}} \triangleq \mathbf{\Omega}-\mathbf{\Omega}_{r}$. Then, using (5c) and $5 \mathrm{~d}$ ) one obtains the following coupled error dynamics:

$$
\begin{aligned}
\mathbf{M} \dot{\overrightarrow{\mathbf{V}}}+\mathbf{D}^{\top} \dot{\widetilde{\mathbf{\Omega}}}= & \left(\mathbf{M V}+\mathbf{D}^{\top} \mathbf{\Omega}\right)_{\times} \widetilde{\mathbf{\Omega}}+\left(\mathbf{M} \widetilde{\mathbf{V}}+\mathbf{D}^{\top} \widetilde{\mathbf{\Omega}}\right)_{\times} \boldsymbol{\Omega}_{r} \\
& +\mathbf{F}_{g b}+\overline{\mathbf{F}}_{d}+\Delta_{F}+\mathbf{F}_{r}+\mathbf{F}_{c} \\
\mathbf{J} \dot{\widetilde{\mathbf{\Omega}}}+\mathbf{D} \dot{\overrightarrow{\mathbf{V}}}= & (\mathbf{J} \boldsymbol{\Omega}+\mathbf{D V})_{\times} \widetilde{\mathbf{\Omega}}+\left(\mathbf{M V}+\mathbf{D}^{\top} \mathbf{\Omega}\right)_{\times} \widetilde{\mathbf{V}} \\
& +(\mathbf{J} \widetilde{\boldsymbol{\Omega}}+\mathbf{D} \widetilde{\mathbf{V}})_{\times} \boldsymbol{\Omega}_{r}+\left(\mathbf{M} \widetilde{\mathbf{V}}+\mathbf{D}^{\top} \widetilde{\mathbf{\Omega}}\right)_{\times} \mathbf{V}_{r} \\
& +\boldsymbol{\Gamma}_{g}+\boldsymbol{\Gamma}_{d}+\Delta_{\Gamma}+\boldsymbol{\Gamma}_{r}+\boldsymbol{\Gamma}_{c}
\end{aligned}
$$

where $\mathbf{F}_{r}$ and $\boldsymbol{\Gamma}_{r}$, the feedforward terms that should be compensated for by the controller, are defined by:

$$
\begin{aligned}
& \mathbf{F}_{r} \triangleq-\mathbf{M} \dot{\mathbf{V}}_{r}-\mathbf{D}^{\top} \dot{\boldsymbol{\Omega}}_{r}+\left(\mathbf{M V} \mathbf{V}_{r}+\mathbf{D}^{\top} \boldsymbol{\Omega}_{r}\right)_{\times} \boldsymbol{\Omega}_{r} \\
& \boldsymbol{\Gamma}_{r} \triangleq-\mathbf{J} \dot{\boldsymbol{\Omega}}_{r}-\mathbf{D} \dot{\mathbf{V}}_{r}+\left(\mathbf{J} \boldsymbol{\Omega}_{r}+\mathbf{D} \mathbf{V}_{r}\right)_{\times} \boldsymbol{\Omega}_{r}+\left(\mathbf{M V} \mathbf{V}_{r}+\mathbf{D}^{\top} \boldsymbol{\Omega}_{r}\right)_{\times} \mathbf{V}_{r}
\end{aligned}
$$

For the sake of completeness, the following proposition are recalled from our prior work Krupínski et al. (2017).

Proposition 2. (see (Krupínski et al., 2017, Pro.3)) Consider the system dynamics (23a)-23b) and apply the following controller:

$$
\left\{\begin{aligned}
\mathbf{F}_{c}= & -\mathbf{K}_{V} \widetilde{\mathbf{V}}-\mathbf{K}_{i V} \mathbf{z}_{V}-\left(\mathbf{M} \widetilde{\mathbf{V}}+\mathbf{D}^{\top} \widetilde{\mathbf{\Omega}}\right) \times \mathbf{\Omega}_{r} \\
& +\mathbf{D}^{\top}\left(\widetilde{\mathbf{\Omega}} \times \mathbf{\Omega}_{r}\right)+\mathbf{M}\left(\widetilde{\boldsymbol{\Omega}} \times \mathbf{V}_{r}\right)-\mathbf{F}_{g b}-\overline{\mathbf{F}}_{d r}-\mathbf{F}_{r} \\
\boldsymbol{\Gamma}_{c}= & -\mathbf{K}_{\Omega} \widetilde{\boldsymbol{\Omega}}-\mathbf{K}_{i \Omega} \mathbf{z}_{\Omega}-(\mathbf{J} \widetilde{\mathbf{\Omega}})_{\times} \mathbf{\Omega}_{r}-\left(\mathbf{D}^{\top} \widetilde{\mathbf{\Omega}}\right)_{\times} \mathbf{V}_{r} \\
& -\boldsymbol{\Gamma}_{g}-\overline{\boldsymbol{\Gamma}}_{d r}-\boldsymbol{\Gamma}_{r}
\end{aligned}\right.
$$

with $\mathbf{K}_{V}, \mathbf{K}_{\Omega}, \mathbf{K}_{i V}, \mathbf{K}_{i \Omega}$ some positive diagonal $3 \times 3$ gain matrices, $\mathbf{z}_{V} \triangleq \int_{0}^{t} \widetilde{\mathbf{V}}(s) d s, \mathbf{z}_{\Omega} \triangleq \int_{0}^{t} \widetilde{\boldsymbol{\Omega}}(s) d s$, and

$$
\left\{\begin{array}{l}
\overline{\mathbf{F}}_{d r} \triangleq-\left(\mathbf{D}_{V l}+|\mathbf{V}| \mathbf{D}_{V q}\right) \mathbf{V}_{r} \\
\boldsymbol{\Gamma}_{d r} \triangleq-\left(\mathbf{D}_{\Omega l}+|\mathbf{\Omega}| \mathbf{D}_{\Omega q}\right) \boldsymbol{\Omega}_{r}
\end{array}\right.
$$

Assume that the disturbance terms $\Delta_{F}$ and $\Delta_{\Gamma}$ are constant and that $\mathbf{V}_{r}, \mathbf{\Omega}_{r}$ and their derivative are bounded. Then, the equilibrium of the controlled system $\left(\mathbf{V}, \mathbf{\Omega}, \mathbf{z}_{V}, \mathbf{z}_{\Omega}\right)=\left(\mathbf{V}_{r}, \mathbf{\Omega}_{r}, \mathbf{z}_{V}^{\star}, \mathbf{z}_{\Omega}^{\star}\right)$, with $\mathbf{z}_{V}^{\star}=\mathbf{K}_{i V}^{-1} \boldsymbol{\Delta}_{F}$ and $\mathbf{z}_{\Omega}^{\star}=\mathbf{K}_{\Omega}^{-1} \boldsymbol{\Delta}_{\Gamma}$, is globally asymptotically stable (GAS) and locally exponentially stable (LES). 
The proof of this proposition given in Krupínski et al. (2017) consists in showing that the time-derivative of the following Lyapunov function candidate is negative semi-definite:

$$
\begin{aligned}
\mathcal{L}_{\text {inner }} \triangleq \frac{1}{2} \widetilde{\mathbf{W}^{\top}} \mathbf{M}_{T} \widetilde{\mathbf{W}} & +\frac{1}{2}\left(\mathbf{z}_{V}-\mathbf{K}_{i V}^{-1} \boldsymbol{\Delta}_{F}\right)^{\top} \mathbf{K}_{i V}\left(\mathbf{z}_{V}-\mathbf{K}_{i V}^{-1} \boldsymbol{\Delta}_{F}\right) \\
& +\frac{1}{2}\left(\mathbf{z}_{\Omega}-\mathbf{K}_{i \Omega}^{-1} \boldsymbol{\Delta}_{\Gamma}\right)^{\top} \mathbf{K}_{i \Omega}\left(\mathbf{z}_{\Omega}-\mathbf{K}_{i \Omega}^{-1} \boldsymbol{\Delta}_{\Gamma}\right)
\end{aligned}
$$

with $\mathbf{M}_{T}>0$ given by (1) and $\widetilde{\mathbf{W}} \triangleq\left[\widetilde{\mathbf{V}}^{\top}, \widetilde{\mathbf{\Omega}}^{\top}\right]^{\top}$.

In practice the roll motion may not be actuated by conception (i.e. $\Gamma_{c 1} \equiv 0$ ) like the Girona-500 AUV used for experiment validations and is, thus, left passively stabilized by restoring and dissipative roll moments. A solution to such a situation can be easily adapted as proposed in (Krupínski et al. 2017. Sec.IV.A).

\section{Validation results}

\subsection{Comparative simulation results}

This section illustrates the performance of the proposed approach compared to the state-of-the-art IBVS approach proposed in Rives and Borrelly (1997) via a realistic simulation of a fully-actuated AUV model. Simulations have been carried out using Matlab/Simulink. The physical parameters of the simulated fully-actuated AUV given in Tab. 1 are those of the Girona-500 AUV along with rough estimates of added mass,

\begin{tabular}{|c|c|}
\hline Specification & Numerical value \\
\hline Mass $m[k g]$ & 160 \\
\hline $\mathbf{F}_{g b}[N]$ & $1.047 \mathrm{mg}$ \\
\hline $\mathbf{r}_{G}[m]$ & {$\left[\begin{array}{lll}0 & 0 & 0.15\end{array}\right]^{\top}$} \\
\hline $\mathbf{r}_{C}[m]$ & $\left.\begin{array}{ll}0 & 0\end{array}\right]^{\top}$ \\
\hline & $\begin{array}{ll}5 & 10\end{array}$ \\
\hline $\mathbf{J}=\mathbf{J}_{0}+\mathbf{M}_{A}^{22}\left[k g . m^{2}\right]$ & $\begin{array}{lll}5 & 110 & 8\end{array}$ \\
\hline & 10 \\
\hline & {$[20$} \\
\hline $\mathbf{M}_{A}^{11}[k g]$ & $5 \quad 320$ \\
\hline & $10 \quad 12$ \\
\hline & {$\left[\begin{array}{ll}1 & 10\end{array}\right.$} \\
\hline $\mathbf{M}_{A}^{12}=\mathbf{M}_{A}^{21 \top}[k g . m]$ & 101 \\
\hline & $\left.\begin{array}{lll}4 & 3 & 0.5\end{array}\right]$ \\
\hline $\mathbf{D}_{V l}\left[k g . s^{-1}\right]$ & $\operatorname{diag}(1,1.2,1.4)$ \\
\hline $\mathbf{D}_{V q}\left[k g \cdot m^{-1}\right]$ & $\operatorname{diag}(30,1700,2550)$ \\
\hline $\mathbf{D}_{\Omega l}\left[\mathrm{~kg} \cdot \mathrm{m}^{2} \cdot \mathrm{s}^{-1}\right]$ & $\operatorname{diag}(0.3,0.2,0.4)$ \\
\hline $\mathbf{D}_{\Omega q}[N . m]$ & $\operatorname{diag}(3,2,4)$ \\
\hline
\end{tabular}
added inertia and damping coefficients.

Table 1: Specifications of the simulated AUV.

For all comparisons, the value of $\mathbf{q}^{\star}$ is $\mathbf{q}^{\star}=1.9901 \mathbf{e}_{2}$ that corresponds to the situation where the vehicle moves at $1[\mathrm{~m}]$ above and in the middle of the pipeline having a diameter of $0.2[\mathrm{~m}]$. The desired speed $v_{r}$ along the pipeline is $1[\mathrm{~m} / \mathrm{s}]$.

In order to make fair and simple comparisons between the two approaches, it is considered that the current velocity is equal to zero and that the estimated parameters of the AUV's model are equal to the real values. In the sequel, it is called:
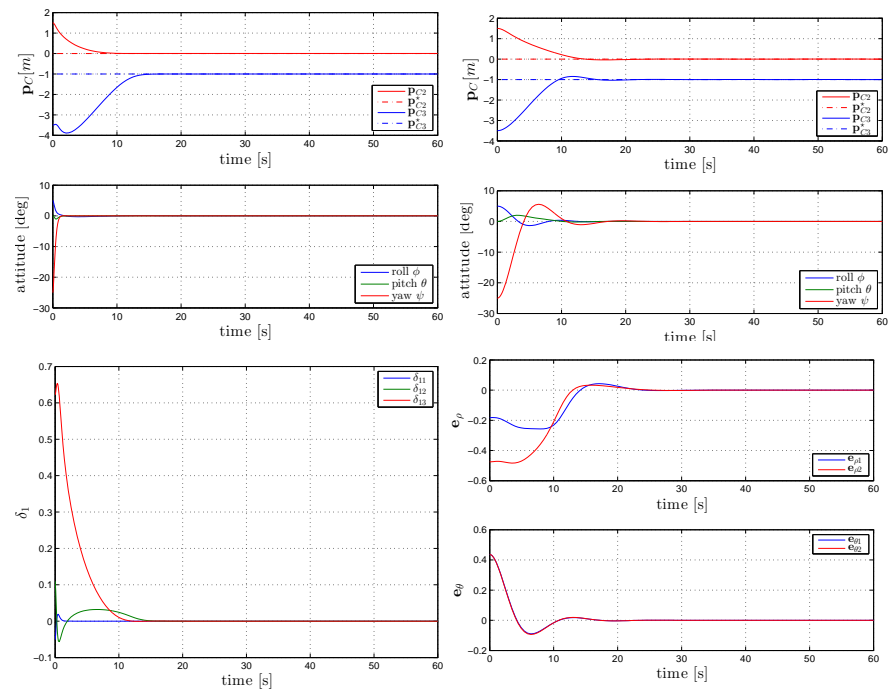

Figure 5: Comparison 1 (left (resp. right) column for controller 1 (resp. 2)) for small initial errors $\mathbf{p}_{C}(0)=[0,1.5,-3.5]^{\top}$ and $\mathbf{R}(0)=\mathbf{R}_{\left\{\frac{5 \pi}{180}, 0, \frac{-25 \pi}{180}\right\}}$ (from top to bottom): AUV position and attitude (Euler angles) vs. time, visual error vs. time.
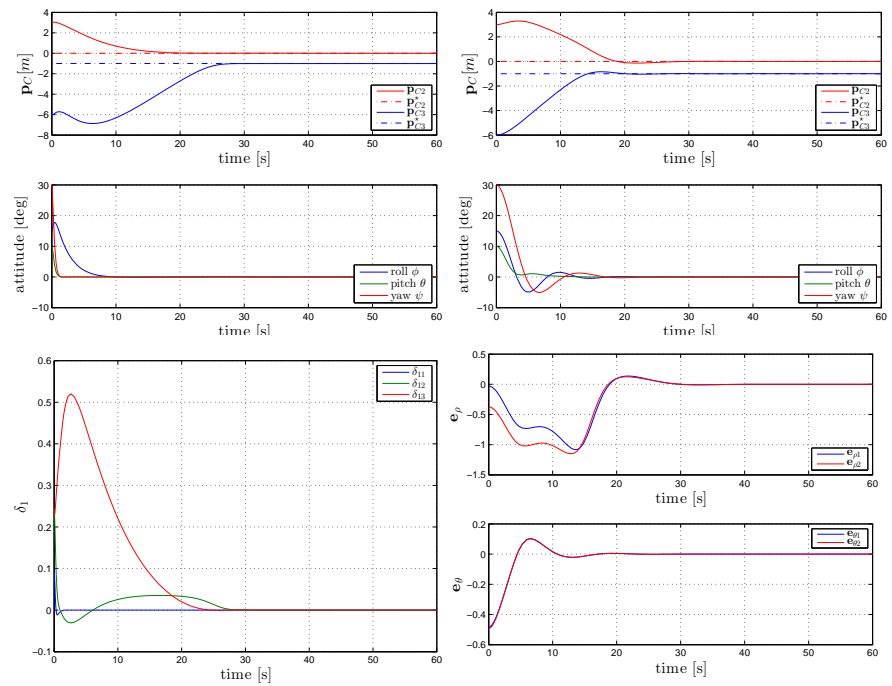

Figure 6: Comparison 2 (left (resp. right) column for controller 1 (resp. 2)) for medium initial errors $\mathbf{p}_{C}(0)=[0,3,-6]^{\top}$ and $\mathbf{R}(0)=\mathbf{R}_{\left\{\frac{15 \pi}{180}, \frac{10 \pi}{180}, \frac{30 \pi}{180}\right\}}$ (from top to bottom): AUV position and attitude (Euler angles) vs. time, visual error vs. time.

- Controller 1 - the proposed controller: The control gains of the inner-loop and outer-loop are tuned based on the classical pole placement technique. For the inner-loop, two triple negative real poles equal to -2 and -4 are chosen for the linearized closed-loop system (24) for the particular case where $\mathbf{V}_{r} \equiv \mathbf{\Omega}_{r} \equiv \mathbf{v}_{f} \equiv \mathbf{0}$. The gain matrices $\mathbf{K}_{V}, \mathbf{K}_{\Omega}, \mathbf{K}_{i V}$ and $\mathbf{K}_{i \Omega}$ are given by

$$
\begin{aligned}
& -\mathbf{K}_{V}=\operatorname{diag}(330.8,922.1,960), \mathbf{K}_{i V}=\mathbf{0}, \\
& -\mathbf{K}_{\Omega}=\operatorname{diag}(351.8,438.7,280), \mathbf{K}_{i \Omega}=\mathbf{0} .
\end{aligned}
$$

For the outer-loop, negative real poles $(-2.6,-1.2)$ for the subsystem of vertical motion, and negative real double pole -2.5 for the subsystem of the lateral motion are used 

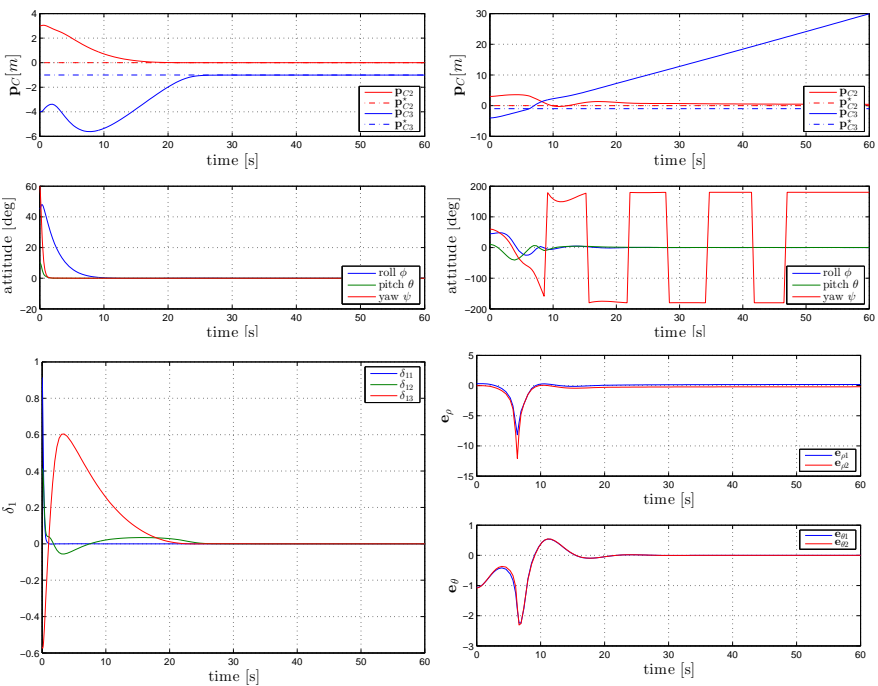

Figure 7: Comparison 3 (left (resp. right) column for controller 1 (resp. 2)) for large initial errors $\mathbf{p}_{C}(0)=[0,3,-4]^{\top}$ and $\mathbf{R}(0)=\mathbf{R}_{\left\{\frac{45 \pi}{180}, \frac{10 \pi}{180}, \frac{60 \pi}{180}\right\}}$ (from top to bottom): AUV position and attitude (Euler angles) vs. time, visual error vs. time.

on the linear approximation of system 20, at the equilibrium. The gains are given by

$$
\begin{aligned}
& -k_{u}=6, k_{\omega}=0.5, \\
& -\mathbf{K}_{1}=\operatorname{diag}(41.36,3.31), \mathbf{K}_{2}=\operatorname{diag}(3.8,5), \\
& -\mu\left(\left|U_{1}\right|\right)=\varepsilon+(1-\varepsilon)\left|U_{1}\right|^{2 n} \text { with } \varepsilon=0.05, n=5 .
\end{aligned}
$$

- Controller 2 - the IBVS controller proposed in Rives and Borrelly (1997): It is based on classical visual servoing approach applied to lines, corresponding to the projection of the borderlines of the pipe onto image features. In this case, the visual errors are given by the difference of the polar coordinates $\left[\rho_{1}, \theta_{1}, \rho_{2}, \theta_{2}\right]^{\top}$ of the current lines and the associated values $\left[\rho_{1}^{\star}, \theta_{1}^{\star}, \rho_{2}^{\star}, \theta_{2}^{\star}\right]^{\top}$ of the desired lines. As discussed in Rives and Borrelly (1997) two lines are not enough to ensure a global minimum, roll stabilization to zero is needed independently. Therefore, the term $k_{\omega} \mathbf{e}_{1}^{\top}\left(\mathbf{e}_{3} \times \mathbf{R}^{\top} \mathbf{e}_{3}\right)$ is added in the computation of the control torques to help the roll stabilization to zero. The gains involved in this controller are $k=0.3, \mu=5 / 3, \beta=1$, $k_{\omega}=0.5$.

Extensive simulations have been carried out using the two controllers. Three simulations are reported next that correspond to three different initial conditions (i.e. small, medium and large errors in translations and rotations).

- In the first simulation (see Fig. 5), both the controllers exhibit a quite good behaviour. The pose (i.e. position and orientation) quickly converges to the desired values while the visual errors converge smoothly to zero.

- In the second simulation (see Fig. 6, the convergence of the visual errors to zero and of the pose to the desired val-

\footnotetext{
${ }^{1}$ The notation of gains $k, \mu, \beta$ is adopted in Rives and Borrelly (1997)
}

ues is still achieved for both controllers. However, one observes some oscillations in the attitude's time evolution of Controller 2 in contrast to the smooth convergence without overshoot in the attitude of Controller 1.

- When the initial errors are very large especially in roll angle (see Fig. 7), Controller 2 becomes unstable while Controller 1 still ensures a very satisfactory performance (i.e. fast convergence without oscillations). The poor performance of Controller 1 in this case is not surprising since its design and stability analysis are only established on local basis.

The reported simulations show some net improvements in terms of convergence domain and smooth transient response of the proposed IBVS approach w.r.t. to the IBVS approach proposed in Rives and Borrelly (1997).

\subsection{Experimental results}

The Girona-500 AUV developed by the Underwater Vision and Robotics Center (Girona, Spain) Ribas et al. (2012) (see Fig. 8) has been used to perform experimental validations. The AUV is composed of an aluminium frame to support three torpedo-shaped hulls. Its dimensions are $1 \times 1 \times 1.5[\mathrm{~m}]$ in height, width and length, and its weight is approximately $160[\mathrm{~kg}]$ in air. The vehicle is actuated by two horizontal thrusters for yaw and surge actuations, two vertical thrusters for heave and pitch actuations and one lateral thruster for sway actuation. Roll motion is left passively stabilized (i.e. $\Gamma_{c 1} \equiv 0$ ). The mounted sensor suite of the AUV consists of an IMU, a DVL and a downwardlooking camera providing images at about 5-7[ $\mathrm{Hz}]$.
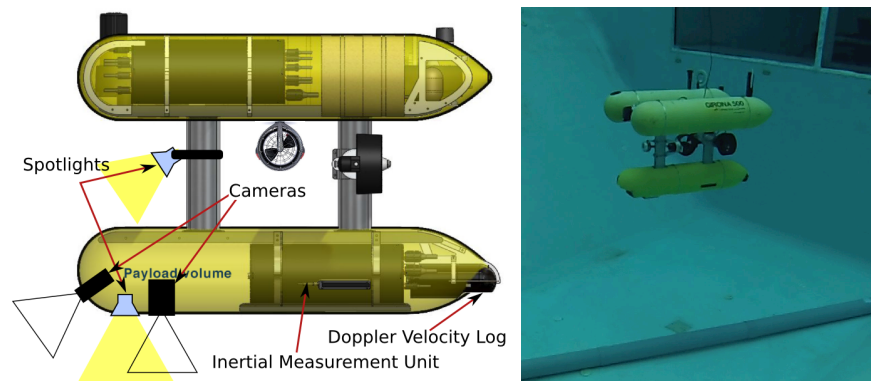

Figure 8: Girona-500 AUV and experimental setup

In order to emulate an inspection of an underwater pipeline, a pipeline mockup, whose diameter is approximatively $0.2[\mathrm{~m}]$, is placed in a pool (see Fig. 8). ROS middleware is used to transfer images from camera in low-bandwidth compressed formats. A bridge between ROS images and OpenCV is also used to obtain in real time the parameters of the pipeline borders.

The control gains and other parameters involved in the computation of the control inputs are given by

- $\mathbf{K}_{V}=\operatorname{diag}(145.4,418.5,480), \mathbf{K}_{i V}=0.1 \mathbf{K}_{V}$

- $\mathbf{K}_{\Omega}=\operatorname{diag}(96.9,124.7,70), \mathbf{K}_{i \Omega}=0.1 \mathbf{K}_{\Omega}$

- $k_{u}=0.5, k_{\omega}=1$

- $\mathbf{K}_{1}=\operatorname{diag}(2.65,0.3), \mathbf{K}_{2}=\operatorname{diag}(0.9,1.5)$ 


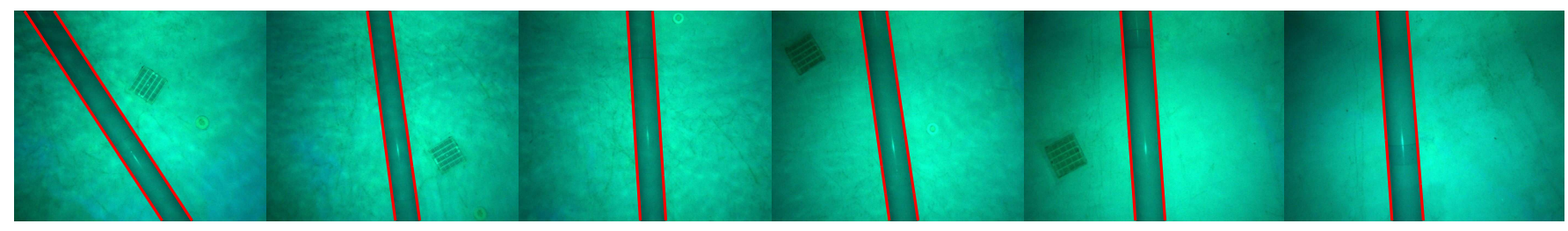
(a) Initial image
(b) $t=2 s$
(c) $t=5 \mathrm{~s}$
(d) $t=12 \mathrm{~s}$
(e) $t=19 \mathrm{~s}$
(f) $t=26 \mathrm{~s}$

Figure 9: Initial image and current images during convergence

- $\mathbf{q}^{\star}=1.9901 \mathbf{e}_{2}, v_{r}=0.15[\mathrm{~m} / \mathrm{s}]$

- $\mathbf{r}_{C}=[0.5,0,0.5]^{\top}[m]$

The estimated summed inertia (i.e., inertia + added inertia) and summed mass (i.e., mass + added mass) are those in Tab. 1 in which the off-diagonal elements are neglected. Finally, the damping force and torque vectors (i.e. $\overline{\mathbf{F}}_{d}$ and $\boldsymbol{\Gamma}_{d}$ ) are also neglected. In the following, experimental results will be reported. Due to space limitation, only brief but representative parts of total results are presented. However, the reader is invited to view a video clip showing the whole experiment (see multimedia attachment) at https: //youtu . be/jPHIJ2CYHLI.
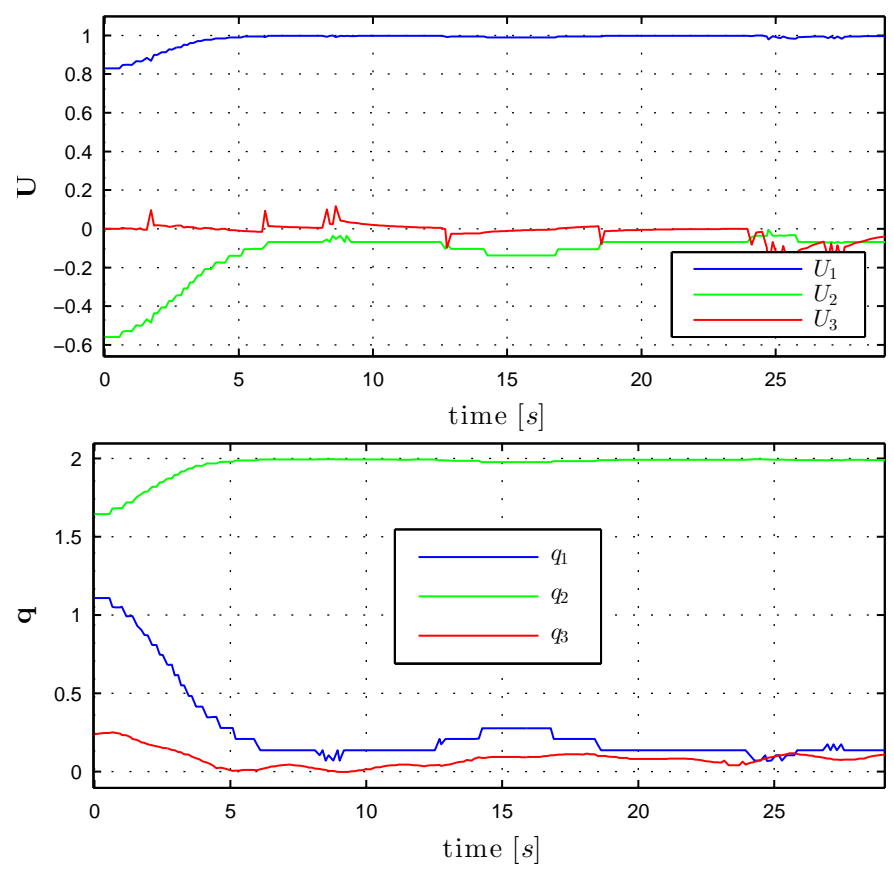

Figure 10: Direction of the pipeline and vector $\mathbf{q}$ vs. time

Experimental results are reported in Figs. 9 - 12 . They correspond to the multimedia attachment. Fig. 10 shows the practical convergence of $\mathbf{U}$ near to $\mathbf{e}_{1}$ whereas the vector of image feature $\mathbf{q}$ converges near to the desired value. The time evolution of the visual error $\delta_{1}$ is given in Fig. 11. One observes that the convergence is obtained in a short period with quite satisfactory behaviour. Fig. 9 presents an overview of current images taken during the AUV's motion where the lines obtained from image processing using Hough algorithm are displayed in red.

Finally, Fig. 12 shows the control force and torque vectors computed from the inner-loop. Since the Girona-500 AUV is

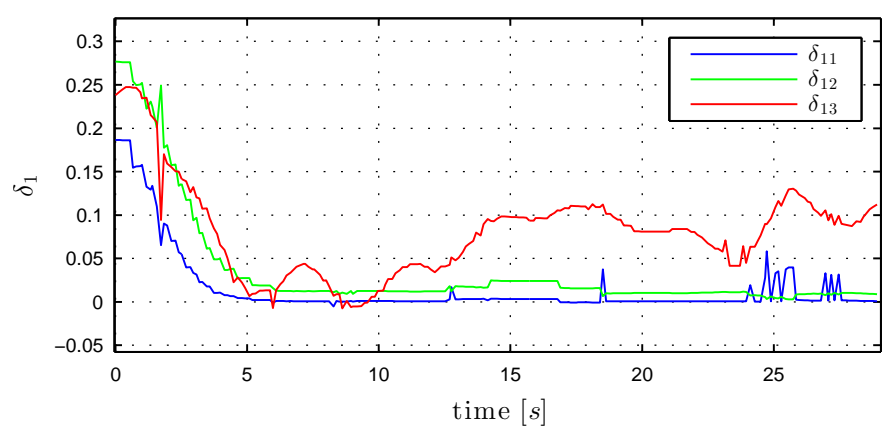

Figure 11: Visual error $\delta_{1}$ vs. time
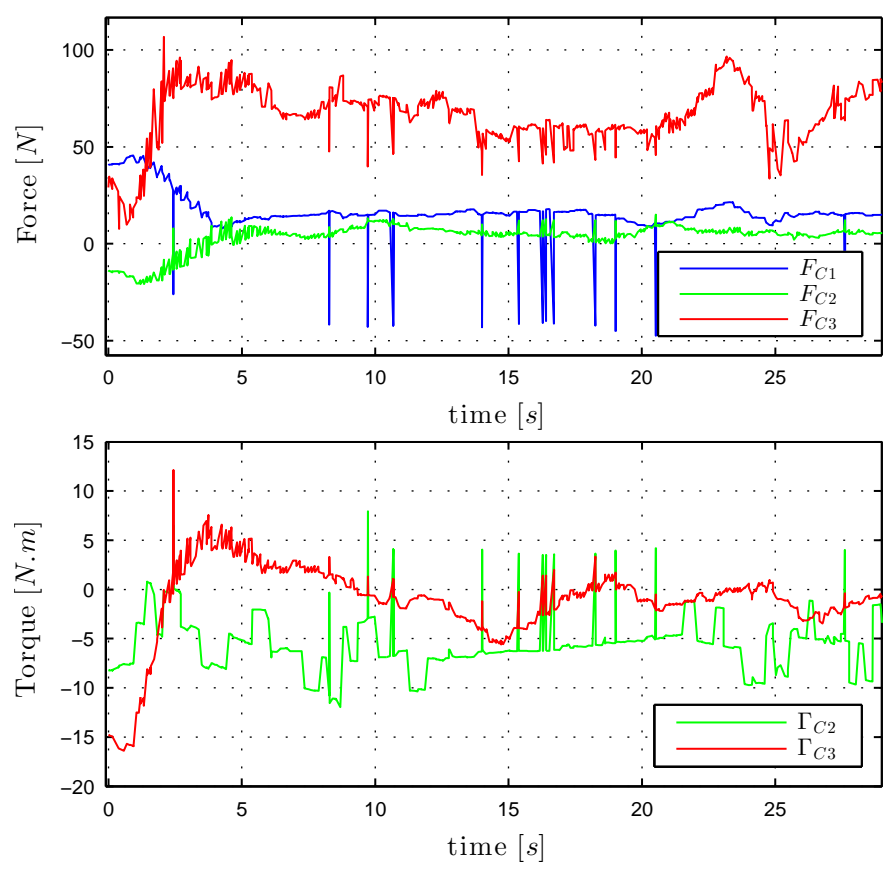

Figure 12: Control force and torque vs. time

positively buoyant, the third component of the control force vector practically converges to $75[N]$. The longitudinal component $F_{c 1}$ practically converges to the force needed to counteract the drag force corresponding to the forward velocity about $0.15[\mathrm{~m} / \mathrm{s}]$ along the pipeline. The lateral component $F_{c 2}$ also converges near to a non-null value $(\approx 6[N])$ which can be explained by the fact that the vehicle is not perfectly aligned with the pipeline and thus resulting in non-negligible lateral drag. As for the control torque, the second component $\Gamma_{c 2}$ converges near to $-7[N . m]$, allowing to maintain the vehicle horizontally. This non-null value is due to the fact that the vector $\overrightarrow{B G}$ connecting 
the $\mathrm{CoB}$ and $\mathrm{CoM}$ is not aligned with the vertical basis vector $\vec{e}_{3}^{b}$. From this figure one also observes some isolated spikes in the control forces and torques. This is intrinsically due to the fact that for some "security" reason the Girona-500 AUV randomly sent some impulsive additive signals to the inner-loop control independently from the proposed controller. However, these "incidental" random and short signals did not affect the overall performance of the proposed approach, showing the robustness of the latter.

\section{Conclusions}

A nonlinear visual servo control for pipeline following for fully-actuated AUVs has been proposed. The originality of the proposed approach lies in exploiting the full system dynamics in control design. The controller directly uses the image features as feedback information without exploiting the relative pose of the vehicle with respect to the environment. Since practically no knowledge of the Cartesian world is mandatory, the implementation, especially in uncertain or changing scenes is greatly simplified. Rigorous stability analysis for closed-loop systems has been given. The theoretical analysis has been complemented by comparative simulation results between the proposed control approach and an existing IBVS controller and also by experimental validations that shows the effectiveness of the proposed control scheme, even when the system parameters are not known precisely. As perspectives, it would be interesting to improve the proposed approach in the case where DVL measurements become inaccurate or missing, due to low velocity or in close proximity to man-made infrastructures.

Acknowledgement: This work was supported by the CNRSPEPS CONGRE project, the FUI GreenExplorer project and by Cybernetix company (Technip group).

\section{References}

Aguiar, A. P., Pascoal, A. M., 2007. Dynamic positioning and way-point tracking of underactuated AUVs in the presence of ocean currents. International Journal of Control 80 (7), 1092-1108.

Allen, B., Stokey, R., Austin, T., Forrester, N., Goldsborough, R., Purcell, M., von Alt, C., 1997. REMUS: a small, low cost AUV; system description, field trials and performance results. In: MTS/IEEE OCEANS'97. pp. 994-1000.

Antich, J., Ortiz, A., 2003. Underwater cable tracking by visual feedback. Pattern Recognition and Image Analysis 2652, 53-61.

Antonelli, G., 2007. On the use of adaptive/integral actions for six-degreesof-freedom control of autonomous underwater vehicles. IEEE Journal of Oceanic Engineering 32 (2), 300-312.

Bagnitsky, A., Inzartsev, A., Pavin, A., Melman, S., Morozov, M., 2011. Side scan sonar using for underwater cables \& pipelines tracking by means of auv. In: IEEE Symposium on Underwater Technology (UT) and Workshop on Scientific Use of Submarine Cables and Related Technologies (SSC). pp. $1-10$.

Christ, R., Wernli, R., 2007. The ROV Manual: A User Guide for Observation Class Remotely Operated Vehicles. Butterworth-Heinemann.

Fossen, T. I., 2002. Marine Control Systems. Marine Cybernetix AS.

Fryxell, D., Oliveira, P., Pascoal, A., Silvestre, C., Kaminer, I., 1996. Navigation, guidance and control of AUVs: An application to the MARIUS vehicle. Control Engineering Practice 4, 401-409.

Inzartsev, A., Pavin, A., 2009. AUV application for inspection of underwater communications. INTECH Open Access Publisher.
Josserand, T. M., 2006. Optimally-robust nonlinear control of a class of robotic underwater vehicles. Ph.d. thesis, University of Texas at Austin.

Khalil, H. K., 2002. Nonlinear systems (3rd Edition). Prentice Hall.

Krupínski, S., Allibert, G., Hua, M., Hamel, T., 2017. An inertial-aided homography-based visual servo control approach for (almost) fully-actuated autonomous underwater vehicles. IEEE Transactions on Robotics 33 (5), 1041-1060.

Krupínski, S., Allibert, G., Hua, M.-D., Hamel, T., 2012. Pipeline tracking for fully-actuated autonomous underwater vehicle using visual servo control. In: American Control Conference (ACC). pp. 6196-6202.

Krupínski, S., Desouche, R., Palomeras, N., Allibert, G., Hua, M.-D., 2015. Pool testing of auv visual servoing for autonomous inspection. In: 4th IFAC Workshop on Navigation, Guidance and Control of Underwater Vehicles, IFAC-PapersOnLine. Vol. 48. pp. 274-280.

Lapierre, L., Soetanto, D., Pascoal, A., 2008. Robust nonlinear path-following control of an AUV. IEEE Journal of Oceanic Engineering 33, 89-102.

Leonard, N. E., 1997. Stability of a bottom-heavy underwater vehicle. Automatica 33 (3), 331-246.

Mahony, R., Hamel, T., 2005. Image-based visual servo control of aerial robotic systems using linear image features. IEEE Transactions on Robotics 21 (2), 227-239.

Matsumoto, S., Yoshihiko, I., 1995. Real-time vision-based tracking of submarine-cables for AUV/ROV. In: MTS/IEEE OCEANS95. pp. $1997-$ 2002.

Naeem, W., Sutton, R., Ahmad, S. M., Burns, R. S., 2003. A review of guidance laws applicable to unmanned underwater vehicles. The Journal of Navigation 56 (1), 15-29.

Plücker, J., 1865. On a new geometry of space. Philosophical Transactions of the Royal Society of London 155, 725-791.

Refsnes, J. E., Sorensen, A. J., Pettersen, K. Y., 2008. Model-based output feedback control of slender-body underactuated AUVs: theory and experiments. IEEE Transactions on Control Systems Technology 16 (5), 930-946.

Repoulias, F., Papadopoulos, E., 2007. Planar trajectory planning and tracking control design for underactuated AUVs. Ocean Engineering 34 (11), 16501667.

Ribas, D., Palomeras, N., Ridao, P., Carreras, M., Mallios, A., 2012. Girona 500 AUV: From survey to intervention. IEEE/ASME Transactions on Mechatronics 1 (17), 46-53.

Rives, P., Borrelly, J.-J., 1997. Underwater pipe inspection task using visual servoing techniques. In: IEEE International Conference on Intelligent Robots and Systems (IROS). pp. 63-68.

Samson, C., Espiau, B., Borgne, M. L., 1991. Robot control: the task function approach. Oxford University Press.

Shukla, A., Karki, H., 2016a. Application of robotics in offshore oil and gas industry a review part ii. Robotics and Autonomous Systems 75, 508 - 524 .

Shukla, A., Karki, H., 2016b. Application of robotics in onshore oil and gas industry-a review part i. Robotics and Autonomous Systems 75, 490-507.

Smallwood, D., Whitcomb, L. L., 2004. Model-based dynamic positioning of underwater robotic vehicles: theory and experiment. IEEE Journal of Oceanic Engineering 29 (1), 169-186.

Spangelo, I., Egeland, O., 1994. Trajectory planning and collision avoidance for underwater vehicles using optimal control. IEEE Journal of Oceanic Engineering 19, 502-511. 